\title{
Espacio y política en el desarrollo territorial
}

\section{Space and politics in territorial development}

\author{
Blanca Rebeca Ramírez-Velázquez*
}

\begin{abstract}
There is a transit in contemporary literature in Mexico from regional to territorial development as part of a new trend regarding categories such as space, region and territory. Then, there is a need to clarify and give sense to said changes within the context of new orientations and possibilities in the generation of contemporary development policies. The aim of this contribution is to analyze the most important changes in territorial development taking into account the redefinition of territory in Latin America and changes in space conceptions. The main point is to open the process to multiple agents and tendencies that social and cultural trends could generate in multidimensional scales and levels.
\end{abstract}

Keywords: territory, development policies, co-presence, co-existence.

\section{Resumen}

El paso que existe en la literatura contemporánea del desarrollo regional al territorial lleva consigo un cambio importante en el significado de las categorías de espacio, región y territorio que es preciso evidenciar y clarificar para dar sentido a las nuevas orientaciones y posibilidades que tiene la política del desarrollo en la época contemporánea. Con el objetivo de contribuir a esta reflexión, se propone analizar los cambios más importantes ocurridos en el análisis sobre el desarrollo territorial a partir de la redefinición que se ha dado de la categoría de territorio en América Latina, abriendo su dimensión a los múltiples agentes que intervienen en el proceso, para integrar una estrategia que pueda redimensionar el desarrollo territorial a partir de la consideración de la multidimensionalidad que éste tiene en sus diferentes niveles y escalas.

Palabras clave: territorio, política de desarrollo, copresencias, coexistencias

* Universidad Autónoma Metropolitana, Xochimilco, México. Correo-e: blare19@prodigy.net. mx, bramirez@correo.uam.mx. 


\section{Introducción}

Durante muchos ańos, una de las preocupaciones fundamentales de los gobiernos de diversos países ha sido cómo pasar de un estadio caracterizado como atraso a otro llamado de desarrollo. Lamentablemente, el significado de estos conceptos han sido motivo de innumerables definiciones, pero el más generalizado es el de modernidad. Para realizar este tránsito, el espacio ha sido de gran importancia, al ser el objeto mismo de la transformación, en conjunto con las sociedades que en éste habitan; a pesar de que, hasta hace poco, este hecho no se reconocía.

En la actualidad esta preocupación se ha matizado, pues la crítica que en la posmodernidad se hizo del concepto de desarrollo, implícita o explícitamente, promovió que la preocupación se centrara en encontrar salidas para contender con las desigualdades sociales y territoriales, resultado del desarrollo capitalista. No obstante, la búsqueda por implantar dicha transformación no ha dejado de ser parte de la agenda política de algunos gobiernos.

En esta búsqueda, la importancia del espacio ha estado subordinada a la del desarrollo y la transformación, es decir, al tiempo. Cada día la dimensión espacial se ha revalorizado, y ha adquirido una relevancia fundamental para comprender los procesos contemporáneos (Ramírez, 2006). Si bien la reconsideración de la dimensión espacial ha sido importante, ésta se ha desarrollado a partir de cambios conceptuales que es necesario destacar. El hecho de convocar a la reflexión sobre estrategias que tiendan a generar una política nacional de desarrollo territorial, sin duda es loable.

Llama la atención que, desde hace poco tiempo, en diversos foros se hable del concepto de desarrollo territorial y no regional (Calva, 2007). Para algunas posturas, como la que aquí se suscribe, significa un cambio epistemológico sustantivo en cómo se está percibiendo el espacio y cómo se le acompaña en su evolución, más que de una simple sustitución de términos. Si bien el concepto de espacio se sustituyó por el de territorio, el de desarrollo no se ha transformado y todavía se le percibe como el elemento fundamental del cambio y la evolución. El concepto desarrollo requiere también una nueva definición que se integre y acompañe hoy a la de territorio.

Con el fin de contribuir a esta reflexión, se propone como objetivo de este ensayo analizar los cambios más importantes que se han dado en el análisis sobre el desarrollo territorial, a partir de cómo se ha redefinido la categoría en América Latina y los países anglosajones, abriendo su dimensión a los múltiples agentes que intervienen en el proceso e integrar una estrategia que pueda redimensionar el desarrollo territorial, a partir de la 
consideración de la multidimensionalidad que éste tiene en sus diferentes niveles y escalas. Para ello, la exposición se ha dividido en tres apartados. En la primera, se definen las líneas fundamentales que han determinado el tránsito de conceptos de espacio y región al de territorio en el ámbito de la planeación y la política, incluyendo una redefinición de desarrollo; la segunda centra el análisis en los nuevos agentes y las instituciones en la planeación; en la tercera, se pone a discusión una propuesta metodológica que permitirá integrar al territorio en una política de desarrollo territorial en el país en diferentes niveles y escalas. En cuanto a la metodología, se ha integrado esta reflexión a partir de la revisión teórica de la bibliografía especializada en el tema del desarrollo regional y de los textos que se encargan, a partir de sus diferentes visiones, del estudio del espacio, la región, el territorio y el lugar (Ramírez, 2007a; López y Ramírez, 2010). Asimismo, se presenta un avance de algunas reflexiones propias que se han hecho acerca de la estrategia del desarrollo territorial, como la que integra el documento de Calva (2007) y el de Ramírez (2003b). Asimismo, hacer una revisión de estrategias utilizadas por otros Estados ha sido una constante que permite integrar la que a continuación se propone.

\section{Espacio y desarrollo en la modernidad}

El análisis sobre el espacio tiene una larga historia en los ámbitos de la reflexión filosófica, de la geografía y de la planificación. En los países anglosajones ha sido intensa, comenzó con los textos de la geografía radical en la década de los años sesenta del siglo xx. La crítica a la geografía positivista (Harvery, 1969) y a la geografía cuantitativa (Massey, 1978) se mezcla con una redireccionalización del pensamiento sobre la región, originada por las posturas de Frémont (1976) y de Tuan (1974), quienes "adscribiéndole a la región el carácter de espacio vivido abierto a las percepciones, sentimientos y emociones" (Ramírez, 2007b: 122), abren la posibilidad de conjuntar dos posibilidades en el desarrollo de la geografía regional actual: "la de adscribirse a la región una dimensión local/particular identificada con una escala pequeña [...] o bien la que le da una connotación escalar mediana", que en la opinión de algunos autores, es la que predominaba en ese entonces (Gómez, 2001: 7). Con ello, y a partir de la literatura anglosajona, la necesidad de discutir y analizar el problema de la región desde su dimensión particular, llevó a algunos autores a estudiar el problema usando la categoría de local, en lugar de regional, y dejando esta categoría para redefinir procesos de escala intermedia (Massey, 1996).

Autores latinoamericanos como Coragio (1987) y Pradilla (1984), en los años ochenta también contribuyeron a la discusión, pues dieron con- 
tenido al concepto de espacio al diferenciarlo de las corrientes espacialistas y neoclásicas (Harvey, 1969) imperantes en ese entonces. La crítica fundamental al uso de este concepto tenía dos vertientes: la primera destacaba la falta de concreción que tenía en relación con los procesos generados, y en la cual el espacio era un ente abstracto difícil de retomar con fines de desarrollo; en la segunda, estos planteamientos respondían a la necesidad de recurrir, desde entonces, a uno diferente para referir al ámbito donde se desarrollaban los procesos del capitalismo de los años setenta, que era la de territorio (Pradilla, 1984).

A pesar de la importancia que se dio a este concepto, el de desarrollo se conjuntaba con la región, definida por Coraggio como "una unidad territorial de homogeneidad relativa, ya sea de las relaciones entre agentes y medios o de ciertos atributos de los mismos" (1989: 89). Pradilla, por su parte, sustituyó totalmente los conceptos de región y espacio por el de territorio, pues los consideraba ideológicos y adscritos a las teorías espaciales. La geografía, por su parte, a partir de los años sesenta aportó una amplia literatura sobre el tema (Bassols, 1967). Su propuesta enfatiza el recurso de la regionalización como un elemento fundamental para contender con la necesidad de modernizar y transformar las regiones atrasadas, a partir de un concepto único de desarrollo, en el cual parecería que todas, independientemente de sus características físicas, sociales o culturales, tenían que transitar hacia una visión única del cambio: el capitalista moderno y tecnificado (Ramírez, 2003). Con ello mejorarían las condiciones económicas y sociales de las regiones y de sus habitantes.

En ambas tradiciones, a pesar de utilizar conceptos diferentes -espacio o lugar en la anglosajona, y territorio en la latinoamericana-, es preciso reconocer el carácter de los comentarios que los debates han originado. En el ámbito de la discusión espacio-región, aun en las corrientes radicales, éstas aparecían como unidades homogéneas (o al menos relativamente) diferenciadas de otras regiones y en general aisladas de los agentes que la habitaban, a pesar de que ya la geografía humanista de los setenta había reflexionado sobre la necesidad de integrar espacio-agente, y lo hicieron a partir de la noción de identidad (Tuan, 1974). El carácter de contenedor que la región tenía les daba una dimensión estática, en la cual era el desarrollo o los agentes aislados del espacio quienes cambiaban. Su dimensión era plana, carente de escalas o de profundidad: era la misma forma como se representaba en los modelos (fueran hexagonales, circulares o numéricos) que las ejemplificaban, como en el caso de la teoría del lugar central de Löch y Chistaller (Ramírez, 2003a, cap. 4).

También existía una desarticulación entre espacios, todavía considerada por algunos como oposición entre el campo y la ciudad, así como entre las regiones que buscaban su transformación aisladas y por sí mismas. 
La desvinculación entre los espacios era entonces otra más de las características que había que agregar a su definición, considerando a las urbes los territorios dinámicos y rectores del proceso de desarrollo del o los procesos, y a las zonas rurales como las atrasadas y premodernas.

Las discusiones que se han desarrollado desde finales del siglo xx y en el presente a partir de textos como el de Massey (2005), han producido cambios importantes, generando una resignificación de conceptos. A pesar de que en la literatura anglosajona esto se adscribe al concepto de espacio, la latinoamericana utiliza el de territorio en tres sentidos. Primero, los conceptos de espacio-tiempo no se pueden utilizar separados ni caracterizar dos procesos diferentes: los territoriales por un lado y los del desarrollo por otro. Segundo, hay autores que, por el contrario, conjuntan los procesos, argumentando que están estrechamente vinculados, lo que es de vital importancia para repensar el futuro y el desarrollo de las naciones. Tercero, otros investigadores incluso proponen la conjunción de las categorías, hablando ya de Tiempo-Espacio (Wallerstein, 1998; May y Thrift, 2001).

Para algunos autores como Massey (2005), la redefinición no se limita a una conjunción de categorías, sino a una dimensión epistemológica diferente que permite caracterizar al espacio de otra manera. Si antes el espacio era el contenedor del movimiento (desarrollo y transformación) sin que cambio alguno ocurriera en sus características, ahora son tiempo y espacio los que se mueven conjuntamente, y por tanto, se transforman en una dualidad cambiante sin opuestos (Massey, 2005; Ramírez, 2006). En este sentido, el espacio, caracterizado por algunos como territorio y no como región, deja de ser un contenedor de recursos, elementos, personas o actividades y constituye parte fundamental de la transformación de agentes y territorios relacionados. Esta dinámica tiene tiempos específicos para llevarse a cabo en cada territorio, pero los dos cambian y se transforman. El movimiento no necesariamente es lineal y en un solo sentido, sino que puede presentar diversidades en dirección y en forma.

Desde la geografía humanista, que en nuestras latitudes tiene importancia hasta finales del siglo xx, a pesar de que su estudio comenzó a partir de los años setenta, hay quienes identifican y resignifican sus conceptos usando ahora el de lugar. Éste es más usado en la literatura anglosajona, y se le adscribe en un cambio de escala local e individual, el carácter de espacio vivido, abierto a percepciones, sentimientos y emociones, como ya lo mencionaron Frémont (1976) y Tuan (1974), en Ramírez (2007b). En esta misma corriente hay autores que priorizan el factor identidad sobre el de acción, desarrollada por Giddens (1984), incorporando la experiencia como cualidad existencial y el sentido de lugar referente a su ser natural, como objeto del mundo (Entrikin, 1991: 6). 
El segundo cambio radica en que, si en el transitar al desarrollo el espacio o la región se consideraban unidades homogéneas, ahora, como resultado de la influencia directa de las discusiones del posmodernismo, la diferencia que prevalece a su interior es una de sus características fundamentales. El reconocimiento de la diversidad dentro de un mismo territorio se ha constituido en el elemento fundamental para dar concreción a la vinculación espacio-sociedad y territorio-agente (Ramírez, 2003a: 122). De esta forma, cada territorio se enlaza de manera específica con los agentes que le son propios y transitan a procesos que cambian conjuntamente con él. En ese sentido, el territorio que incluye a los agentes posibilita la concreción específica de los procesos y relaciones que se realizan entre ellos en cambios que son también diferenciales entre territorios diversos.

El tercer cambio se relaciona con el hecho de que el espacio no sólo tiene movimiento, sino también dinámica. Está producido, usado, apropiado, imaginado y transformado por un conjunto de agentes que no sólo son diferentes en sus condiciones económicas, políticas, sociales y culturales, sino que se ubican territorialmente en distintas escalas y con posicionamientos diversos frente a otros agentes con los cuales pueden compartir el territorio donde se encuentran generando copresencias y coexistencias. Éstas pueden existir sin que haya coincidencias en relación con la forma como se percibe su transformación o uso del mismo territorio. Los agentes están posicionados en el territorio con visiones que pueden coincidir sobre su uso o transformación, pero también pueden no hacerlo. El espacio que antes se veía plano, a partir de las diferentes posiciones que guardan los agentes (que pueden ser de muy diversa índole: personas, grupos sociales, instituciones, gobiernos, etc.) y de sus posibilidades de relación, se abre a una multiplicidad de dimensiones que se articulan conjuntando visiones, direcciones, movimientos y velocidades diferenciales que se encuentran en momentos específicos o, algunas veces, no se encuentran. Desde esta perspectiva, el territorio se percibe como una entidad multidimensional, que reproduce también diversas trayectorias y direcciones de actores o grupos distintos, con movimientos que favorecen articulaciones, convergencias o divergencias. Es una multitud de posibilidades que dependen de la escala y las relaciones que se generan a partir de la posición que guardan los agentes frente a los procesos (Ramírez, 2007a).

En suma, se considera el territorio como una unidad de relaciones que se transforma conjuntamente con los procesos y agentes que se vinculan con él en escalas diferenciales, en las cuales las diferencias internas de agentes y procesos se conjuntan para generar, a veces, visiones diversas del transitar en el mundo que coexisten entre ellos. Asimismo, en palabras de Romero, se constituye también como "recursos, como patrimonio, 
como legado para ser entregado a nuestros descendientes en mejores condiciones en que se ha recibido, como bien público y como ámbito de solidaridad y de equidad" (2001: 29). Estos cambios en la definición de espacio para redefinirlo como territorio o lugar, forzosamente orienta la mirada para contender con una nueva forma de identificar a los agentes directamente responsables de poner en marcha su transformación o cambio, es decir, su desarrollo, y por tanto, a redefinir nuevas estrategias y lineamientos que permitan hacerlo.

\section{Los agentes, las instituciones y el Estado}

A partir de la redefinición de territorio, existe una visión alterna de los agentes directamente responsables de implementar el desarrollo. Durante el periodo del Estado benefactor, se sobrevaloró su responsabilidad adjudicándole exclusivamente a éste la autoría intelectual de la estrategia del cambio, y también la de proporcionar los recursos para su implementación con poco interés de otros agentes para involucrarse. Las visiones alternas no eran escuchadas y los agentes que las apoyaban eran excluidos de las estrategias de transformación seleccionadas. Ahora hay nuevos agentes directamente vinculados con las lógicas de desarrollo, también instituciones diversas que lo favorecen, donde el Estado es una más. La incidencia del sector privado en esta tarea se ha incrementado cambiando su carácter público hacia una dimensión privatizadora de los procesos.

Con el arribo de la globalización neoliberal y la exaltación de la competitividad y del libre mercado como elementos fundamentales para vincular al país con estrategias de comercialización internacionales, se argumenta que el Estado es directamente responsable de la crisis que se presentó en los diversos países capitalistas, por lo que hay una tendencia a retirarse de algunas responsabilidades que tenía en el ámbito territorial (Hiernaux, 1991). El gasto público se ha reducido sustantivamente como parte de las acciones necesarias para resolver la crisis, con lo que algunos autores auguran su desaparición para dejar que la iniciativa privada y sus instituciones se encarguen de las transformaciones globales y nacionales, argumentando el fin de la historia (Fukuyama, 1989; Ohmae, 1995). Asimismo se ha ponderado la posibilidad de que los sujetos, en su calidad de individuos o actores del cambio, fueran los responsables de adoptar las medidas necesarias para inducir su transformación, por el sólo hecho de hacerse eficientes y vincularse con los mercados internacionales (nótese que hay diferencia entre agentes y sujeto, pues tiene un sentido muy distinto al que se usa en este ensayo).

Algunos países más que otros se comprometieron con ese pensamiento: México fue uno de ellos. Sin embargo, la experiencia de la Comunidad 
Económica Europea y de algunos países asiáticos muestra que el Estado, en lugar de desaparecer, continúa más que nunca cumpliendo una serie de funciones de seguridad, bienestar y redistribución, lo que indica que en la actualidad en muchos Estados se debería

Inducir su transformación en una entidad capaz de conducir una inserción más discriminada y selectiva en la comunidad internacional; atender y compensar los efectos negativos de la competencia externa en los grupos sociales y territorios más vulnerables; regular los servicios privatizados; y erigirse en una institución social con la aptitud de analizar y atender las demandas económicas y políticas de la población (Moncayo, 2004: 23).

Además, han surgido nuevos agentes del desarrollo que en conjunto establecen reglas que articulan y organizan las interacciones económicas, sociales y políticas entre los individuos, los grupos sociales y el Estado, y que tienen una base geográfica específica. Citando a Terhorst (2004: 13), Rosique los identifica como estructuras desconcentradas del Estado, que se organizan a partir de espacios de compromisos entre los grupos sociales, nacionales, como la competencia en materia de organización del sistema electoral, del régimen fiscal y del régimen provincial (Rosique, 2007: 1). En estos agentes hay que incluir los acuerdos entre pueblos y ciudades para permitir que su gobierno -o bien otros como los que existen en las ONG, tan expandidas en el ámbito social nacional y otras que se presentan en escalas diferenciadas, ya sean globales o locales entre otras- los reconozcan y evidencien.

En esta idea de conjuntar Estado, instituciones y otros agentes sociales, el territorio cuenta con una red muy compleja de integrantes que es posible que tengan visiones conjuntas de proyectos, pero que también pueden confrontarse generando ideas alternas de cambio. Por ejemplo, la oposición que existe entre los defensores de la globalización, quienes la rechazan tanto como los globalifóbicos, o los que argumentan que es preciso generar otra que permita realmente la integración de redes complejas de visiones, a partir de la inclusión en lugar de la exclusión.

Entonces, el reto consiste en articular la copresencia de múltiples agentes que pueden ser individuos, grupos o instituciones, que se ubican en diferentes escalas -internacional, nacional y local- para plantear una visión de desarrollo que las integre, desde una perspectiva incluyente de agentes y territorios, para que cada uno encuentre su lugar en la reproducción social que se pretende construir. Para lograr esta reorganización, la definición de una estrategia de política territorial requiere conocer las ideas de los diferentes agentes (individuos, grupos e instituciones, junto con el Estado) para así favorecer la construcción de redes de vinculación e inclusión, que obedezcan a sus necesidades específicas. En el reconoci- 
miento y encuentro de estas demandas básicas distintas de lugares y agentes, se podrá integrar una visión de futuro nacional, que partirá del reconocimiento de esas diferencias, y no de conformar una mirada única de futuro.

A partir de esta perspectiva, el desarrollo territorial se conforma a partir de diferentes agentes hacedores de política y de estrategia territorial que articulan las distintas visiones: las dominantes del libre mercado internacional con otras que priorizan los mercados locales o regionales o el autoconsumo; las del mantenimiento y preservación de tradiciones y costumbres, que se articulan en redes de movimientos y direcciones diferentes que se caracterizan por la inclusión y la justicia, como elementos fundamentales del nuevo proyecto. Pero, ¿cómo se pueden materializar estas ideas en una estrategia de desarrollo territorial? ¿Cómo generar un nuevo paradigma de desarrollo que considere el territorio como un elemento explicativo esencial de los procesos de transformación y cambio de la sociedad y de los individuos? A continuación se intenta darles respuesta.

\section{Estrategias de desarrollo territorial en una estructura nacional}

A pesar de la creciente ola de neoliberalismo que ha surgido desde la égida de la globalización y del abandono de las concepciones de desarrollo regional y latinoamericano, en la actualidad se retoma la importancia de trabajar con la transformación territorial del país, así como la participación del Estado como director de las estrategias de desarrollo previamente identificadas (Moncayo, 2004).

Si se acepta que existen diferentes ideas de desarrollo, es necesario preguntar: ¿cómo se puede generar un nuevo paradigma que considere el territorio como un elemento esencial de los procesos de transformación y cambio de la sociedad y de los individuos a partir de esas ideas? ¿ Se puede eliminar la concepción binaria que contrapone territorios: unos ganan y otros pierden? ¿A partir de las condiciones, recursos, cultura y tradiciones de cada lugar se puede integrar una visión de desarrollo de estrategias exitosas y diversas? ¿Cómo integrar enfoques de desarrollo humano, sostenible, competitividad sistémica y economía neoinstitucional y considerarlos fundamentales para el desarrollo territorial? (Moncayo, 2004: 17).

Para responder estas preguntas se debe partir de una estrategia que integre tres partes: la primera es de corte general, que diseñe un modelo de utopía surgido de las necesidades nacionales, que para imaginarlo considere los recursos y posibilidades existentes; la segunda requiere adecuarla a los objetivos que servirían para apoyarla, y por último, la tercera, de corte meramente metodológico, necesita el manejo de tres principios 
para asegurar la inclusión de la dimensión territorial en su desarrollo y proporcionar las bases materiales para alcanzar los objetivos diseñados.

En la modernidad, y ahora en la globalización, se han generado visiones de futuro que identifican el desarrollo como un proceso de cambio lineal, en el cual todos los agentes, naciones y continentes tienen que adaptarse a la misma forma de transformación y cambio. En la nueva visión se plantea un camino a la inversa. La transformación no será una y única, sino se articulará aceptando la diversidad y la diferencia como elementos centrales de su estrategia. Es decir, construir una idea que conjunte las alternativas y las diferentes direcciones que el movimiento en el proceso identifique como viables. Se parte del concepto de que ahora el desarrollo no tiene una visión homogénea, sino que el proceso de transformación se construye integrando diferencialmente a los diversos agentes involucrados (Ramírez, 2007a: 131). Asimismo, se propone asumir juicios de valor que son indispensables en la planificacion contemporánea y que responden a preguntas como: ¿quiénes se benefician directamente de esta estrategia o visión? y ¿cómo puede implementarse justamente en cada lugar?

En la segunda, se plantea un ejercicio que identifica los objetivos que marcan directamente los propósitos que se quieren obtener. A continuación se proponen tres para la discusión, que no excluyen otros, pues se consideran de gran importancia.

\subsection{Cohesión territorial económica, social y política sobre la base de enlace justo de copresencias y coexistencias}

Tradicionalmente, el análisis del desarrollo regional se basó en la búsqueda de la eliminación de los desequilibrios sociales y territoriales. En esta propuesta se asume que el desarrollo es diferencial, y que las diferencias y diversidades generadas por la transformación no se eliminarán. Pero se trata de buscar una transición que integre agentes y territorios, eliminando las brechas tan amplias que existen. El factor distributivo es de gran importancia para alcanzar una cohesión social y territorial más incluyente (Ramírez, 2007a: 130). El paso del equilibrio a la cohesión con distribución sería el objetivo central.

La definición de cohesión va dirigida a la búsqueda de caminos distintos, dependiendo de las necesidades, potencialidades y diversidades físicas, naturales y culturales de cada lugar, para eliminar la exclusión de agentes y de territorios de la estrategia de cambio. En este sentido, cohesionar no significa enlazar o unir, sino integrar agentes, territorios e ideas para que cada uno de ellos encuentre su transformación. Se ha enfatizado la necesidad de que ésta sea integral, es decir, que considere todas las 
partes que intervengan en el proceso; e integrante, para que las cobije y dé respuesta a sus necesidades; que sea distributiva, proporcionando a cada uno de acuerdo con sus necesidades y con el trabajo desempeñado para realizarla (Ramírez, 2007a: 130) .

Para ejemplificar, se resalta la importancia que han adquirido los fondos estructurales y de cohesión desarrollados por la Comisión Económica Europea para propiciar la integración territorial de las regiones, lo que ha beneficiado a las regiones periféricas. Se constituyen como instrumento valioso que demuestra la acción de un Estado supranacional en el ejercicio de una búsqueda de integración territorial (Romero, 2001). Parte de identificar los atrasos relativos y desequilibrios territoriales para proporcionar mayor apoyo a las regiones más atrasadas, favoreciendo un cierto reequilibrio territorial sin comprometer los niveles de crecimiento de las regiones centrales. De esta manera, las regiones menos favorecidas situadas en las periferias europeas dispusieron en un contexto institucional de importantes recursos económicos para hacer compatible el doble compromiso de convergencia y cohesión (Romero, 2001: 13). Otro instrumento que permitiría disminuir los efectos negativos generados por una política o una acción, sería el de las compensaciones por efectos negativos; éstos se desarrollarían en forma diversa y requerirían normas específicas para su adjudicación en las estrategias nacionales de cohesión.

\subsection{Uso racional justo y gestión consensada de las bases naturales y del patrimonio natural y cultural}

A partir de la década de los años setenta del siglo xx se ha escrito mucho acerca de la vinculación entre economía y ecología, con el fin de solucionar el abusivo uso de conceptos y visiones antropocéntricas, así como el uso ilimitado de los recursos naturales que ha favorecido su explotación irracional en el mundo. Sin pretender adentrarse en estas discusiones o en las de sostenibilidad y sustentabilidad, se considera necesario integrar la dimensión ambiental de los recursos económicos, que necesariamente tienen una base territorial (Moncayo, 2004: 43-44). Al respecto, se han desarrollado diferentes visiones mediante las cuales se puede analizar la vinculación territorio-ecología-economía, como las de ecorregiones y biorregionalismo.

Más que ampliar estas discusiones, se considera importante identificar cómo se puede gestionar el uso o la transformación de la base natural o cultural de los diferentes territorios. Al responder se podría incluir el desarrollo de dos cualidades: ser racional, que implica coherencia y sensatez en su empleo, o disfrute del acercamiento a los recursos; y ser justo, es decir, que su uso sea honesto, merecido y apropiado. Si se integran estos 
conceptos en la estrategia, la contradicción que existe entre la necesidad de preservar y conservar las bases naturales de los territorios, y la necesidad de algunos grupos de utilizar los recursos que se encuentran en áreas de preservación y conservación, tendría una salida que no necesariamente implique la imposibilidad de seguir usando recursos que les han pertenecido por mucho tiempo, como es el caso de algunos grupos campesinos que ahora viven en zonas de conservación o preservación y ven limitada su posibilidad de uso de los recursos que les pertenecen, y que no pueden seguir explotando para generar su propio desarrollo, porque se ubican en zonas importantes para reproducir a otros, como las ciudades, y que son expuestos a riesgos y, por tanto, se ven en una situación vulnerable.

El uso y apropiación de recursos, el desarrollo sostenible, la gestión prudente y la preservación de la naturaleza y del patrimonio cultural deberían garantizar una transformación equitativa de los entornos locales y una reproducción de la identidad territorial regional (Romero, 2001: 28).

\subsection{Competitividad más equitativa y justa}

En los últimos años, el concepto tradicional de competitividad ha sido cuestionado por muchos autores, que han percibido una obsesión exagerada por imponerlo en los territorios a diferentes escalas. De acuerdo con Moncayo, el mismo Krugman ha criticado la obsesión por la competitividad (2004: 36). Sin embargo, es necesario aceptar la importancia que tiene para generar el proyecto de futuro, y por eso es preciso resignificar esta categoría para darle un contenido en la propuesta.

Contraria a la definición del Fondo Monetario Internacional o la del Banco Mundial, construida a partir del desplazamiento (exclusión) de otro producto, o productor similar, del abaratamiento de los precios, de medidas cambiarias o de reducción de costos de producción generalmente basados en la disminución de salarios (Collin y Cadena, 2007: 332), en 1985 la Comisión Presidencial sobre Competitividad Industrial acuñó una que puede ser más aceptable y sobre todo equitativa. Ésta se concibe como "la capacidad de un país para sostener y expandir su participación en los mercados internacionales y elevar simultáneamente el nivel de vida de su población” (Moncayo, 2004: 32).

Por su parte, la CEPAL también propone una que integra elementos de productividad y progreso técnico (Moncayo, 2004: 33) que conciben una manera de saber hacer, que favorece el desarrollo tecnológico y de las fuerzas productivas, lo que permitiría contender con la flexibilidad productiva contemporánea. El Diamante de Porter y otras propuestas se basan en la necesidad de generar polos de desarrollo tecnológico independientes e innovadores, como parte integrante de la estrategia para hacer 
a los territorios (ciudades o regiones) competitivos (Moncayo, 2004: 3334). Sin embargo, el problema con esta propuesta radica en que se contempla la tecnología como el único elemento que genera desarrollo y transformación de regiones, sin pensar que existen otras que también son importantes y que, con otros parámetros, también pueden generarlo.

¿Cómo cohesionar copresencias y aceptar que hay agentes y actores competitivos en relación con el modelo imperante de la globalización? ¿Cómo aceptar que hay otros cuya competencia no es internacional, sino nacional o regional, que sin ser competitivas, son necesarias para generar y alcanzar una mayor equidad entre los territorios? Éste es el caso de economías campesinas como las de México, que no necesariamente pretenden contender en el mercado internacional, pero sí tener una actividad que les proporcione un mejor ingreso que les permita reproducirse dignamente. En estos casos, el manejo de estrategias que apoyen la permanencia de estas economías en el campo contribuiría a disminuir los movimientos migratorios nacionales e internacionales que realizan los campesinos para subsistir. Ello redundaría en un mayor equilibrio entre los agentes rurales, sin que necesariamente se eliminara la diferencia entre los productores que se integraron al mercado internacional y quienes encontraron otros, nacionales o locales.

Esto implica medir la competitividad a partir de flujos comerciales y estructuras de precios que permiten la introducción al mercado internacional, pero también por el efecto social y territorial que el mantenimiento de agentes originaría en las estructuras productivas y de ingreso de los diferentes mercados y territorios. En este sentido, es posible plantear una definición de beneficio económico, que podría no ser el óptimo o el máximo, o incluso uno no medible desde el punto de vista económico, pero sí a partir del de justicia social. De esta manera, agentes sociales competitivos globalmente podrían coexistir con agentes competitivos distintos, o los que sólo obtienen incremento en su ingreso, aunque no tengan derrama económica importante (Cortina, 2006). Con el simple hecho de aumentar sus ingresos, influirían territorialmente en zonas que son expulsoras de población, y se integrarían a las actividades de su territorio.

También es necesario contar con economías localizadas que puedan adaptarse rápidamente a las transformaciones que se generan en los territorios en la actualidad. Se ha hablado de innovación y de competitividad, pero relacionada con ella está la flexibilidad, que consiste en adaptarse de manera rápida a las necesidades cambiantes de la sociedad. En esto, quizá las economías menos desarrolladas del país han dado cuenta de una mayor adecuación a las necesidades del mercado, con lo cual sería conveniente 
adentrarse en su conocimiento y en los mecanismos institucionales, sobre todo de acuerdos internos entre comunidades, que les han permitido mantenerse y hacerse más flexibles (Cortina, 2006).

Por último, la tercera parte de la estrategia implica agregar tres principios que sería preciso desarrollar. Son elementos de carácter metodológico, específicos para el análisis territorial (Ramírez, 2003a) e indispensables para garantizar que la nueva concepción de espacio quede integrada en su desarrollo.

1) Identificación clara de las diferencias y diversidades existentes en el territorio. Ésta es una parte fundamental en el desarrollo metodológico de la estrategia, ya que a partir de un diagnóstico permite identificar agentes, sus territorios y las formas específicas en que cada uno de ellos se articula en el uso, producción y transformación de su entorno. Con ello se identificarán las visiones y objetivos de cada agente o grupo de agentes, así como las copresencias que evitan al máximo la reducción de ideas sobre un mismo territorio.

2) Vinculación-cohesión de las diferentes visiones a escala de desarrollo que permitan, en sus diferentes niveles, articular estrategias diversas: las urbanas, las regionales, la nacional, la internacional, entre otras. Con esto se pretende generar relaciones más justas, interdependientes y cohesionadas. Éstas tendrían que desagregarse en al menos tres escalas territoriales: primero, los territorios urbanos y rurales para favorecer no solamente las actividades desarrolladas en las zonas urbanas, sino como prioridad geoestratégica las rurales (Ramírez, 2007a). Dentro de ellas, las económicamente redituables y también las campesinas y las de autoconsumo. Segundo, las ciudades en sus diferentes tamaños y funciones $y$, tercero, las regiones.

Lo anterior requiere integrar la visión macro de hacia dónde vamos y cómo lo hacemos. En esta misma escala, es necesario delinear las políticas territoriales tendentes a encontrar el lugar de desarrollo de cada agente, que permita vislumbrar posibilidades de mayor equilibrio para incentivar las actividades productivas en sus diferentes tipos. En la integración de estas ideas que exploran gamas de relaciones sociales corresponde al Estado, en una interacción directa con los agentes ubicados en sus territorios, la integración de visiones y la generación de sinergias, por medio de apoyos específicos que permitan complementar las políticas.

Al mismo tiempo, se tienen que generar proyectos concretos de dimensión urbana, territorial, regional y local para acceder a dichas visiones en sus diferentes dimensiones. La congruencia 
entre proyectos territoriales diversificados y la política de desarrollo general es fundamental para llevarla a cabo. Por ejemplo, fácilmente se podría aceptar la generación de megaproyectos comerciales que integren a los agentes que aceptan este tipo de ideas de desarrollo. El problema radica en que no se alterna con otras opciones, lo que provoca que los lugares que cuestionan su pertinencia no sean aceptados socialmente, dejando de lado la integración de otros agentes y otras visiones que también pudiesen ser vistas como parte del desarrollo.

Por último, esta congruencia debe manifestarse también en la escala individual, donde demandas como salario digno, igualdad de oportunidades entre géneros y edades y vivienda de calidad, entre otras, se mueven en los ámbitos de producción y consumo individuales y se tienen que articular con las otras. La desarticulación de estas utopías (Harvey, 2000), ocasionada por la falta de integración de las visiones de cambio (tiempo) y las exclusiones de espacios/agentes (territorio), es fundamental superarla para conciliar una estrategia de desarrollo territorial exitosa.

¿Cómo cohesionar esas estrategias jerarquizadas que tienen territorios diversos de implementación, y en las cuales diferentes actores forman parte de cada una de ellas? La cooperación interinstitucional entre administraciones públicas, y de éstas con los sectores público y los agentes sociales que habitan los territorios, sin duda es un elemento clave que permite generar sinergias para alcanzar el tercer principio.

3) La integración de relaciones que se basen en esquemas de no exclusión, que generen redes de acceso a diversas posibilidades y alternativas laborales, sociales, culturales e interinstitucionales; de respeto, que posibiliten el acceso equitativo de los agentes a las infraestructuras y al conocimiento en las ciudades y en las regiones, permitiría, sin duda, garantizar nuevas formas de acceso y organización de los territorios del país.

La generación de otras formas de gobierno que coadyuven a la organización de estas nuevas relaciones más horizontales sin duda es importante. Asimismo, es preciso especificar que se habla de vinculación, más no de integración, pues refieren a acciones diferentes. Integrar es un acto que intenta hacer que los territorios se conviertan en una gran familia, generando lazos y ligaduras, mientras que vincular hace que formen 
parte de algo, sin que necesariamente pierdan su identidad o formen una unidad única e indisoluble.

\section{Reflexiones finales}

El cambio de categoría de espacio a territorio tiene varias dimensiones: la de la lengua, donde en la literatura anglosajona la dimensión particular del espacio que es general se concretiza a partir de la de lugar (place), y en la latinoamericana, donde lo hace a partir de la de territorio (López y Ramírez, 2010). En ambos casos y por motivos diferentes, específicamente del lenguaje, tienden a unir los agentes con el espacio donde viven, lo que le quita la dimensión ambigua y general que tiene la de espacio y le da una escala más particular y no intermedia o mediana como la tiene la de región.

Reconocer estas precisiones, así como los usos que se han dado a la de territorio en nuestros entornos, permite afirmar que el uso de la categoría de desarrollo territorial permite hacer una propuesta que vaya más allá de considerar al espacio como mero soporte contenedor de cosas, personas o actividades y lo integre a un proceso de cambio en conjunción con los agentes que lo usan, se lo apropian o lo transforman.

Partir de definiciones claras también posibilita proponer estrategias que permitan hablar de otras categorías que difícilmente se han incluido en las propuestas de desarrollo que se hacen, y solamente se han considerado como dadas, o bien producidas en automático al implementarse la transformación deseada. Se parte de asumir que es preciso tener claro qué tipo de desarrollo se pretende implementar, a qué agentes se quiere beneficiar y quienes serían los directamente responsables de implementar qué tipo de acciones específicas. Así, la búsqueda de distribución y cohesión, respetando las múltiples visiones que se integran, competitividad justa con un uso racional de los recursos, da responsabilidad presente y futura a individuos o grupos sociales, y no deja a la responsabilidad exclusiva del Estado acciones que son implementadas por otros agentes con intereses divergentes.

Existen casos exitosos de estrategias inclusivas de territorios que han generado mejoras en el ingreso económico, disminuyendo así los desequilibrios que el capitalismo globalizador contemporáneo ha incrementado. El caso de la Comunidad Económica Europea es uno de ellos, donde la aplicación, en un medio innovador, de los principios de autonomía política regional, de coordinación y cooperación institucional, de subsidiariedad y de organización de las partes, en un marco de planificación estratégica, han favorecido la compensación de desequilibrios territoriales, la mejora de los niveles de competitividad y el desarrollo del Estado de 
bienestar, elemento fundamental de garantía para mejorar la cohesión social y el crecimiento económico, la carencia histórica más importante de la sociedad (Romero, 2001: 14). A pesar de los beneficios obtenidos, queda mucho por hacer; todavía hay rasgos de exclusión importante que refiere a otros agentes no considerados en las estrategias implementadas, como los migrantes y los extranjeros.

Pero los temas pendientes se hacen más evidentes en países como México, donde a pesar de la posición favorecida que tenemos ante la situación internacional, no se han establecido las estrategias necesarias para lograr dirimir las desigualdades entre regiones, agentes y sociedades, donde la complejidad que presenta el territorio requiere, necesariamente, una creatividad mayor que permita incursionar en formas alternativas para implementar una transformación pacífica y consensada de hacia dónde vamos, y de instrumentos de política pública que organicen el cambio y las diferentes formas de cómo llevarlo a cabo.

\section{Bibliografía}

Bassols-Batalla, Ángel (1967), La división económica regional de México, Universidad Nacional Autónoma de México, México.

Calva, José Luis (coord.) (2007), Agenda de desarrollo 2006-2020, t. XIII, Politicas de desarrollo territorial, Universidad Nacional Autónoma de México-Miguel Ángel Porrúa, México, 17 tomos.

Collin-Harguindeguy, Laura y Félix Cadena Barquin (2007), "Pobreza y exclusión”, en José Luis Calva, Agenda de desarrollo 2006-2020, t. XIII, Politicas de desarrollo territorial, Universidad Nacional Autónoma de México-Miguel Ángel Porrúa, México, pp. 323-349.

Coraggio, José Luis (1989), La cuestión regional en América Latina, International Institute for Environmental Development-América Latina, Quito.

Coraggio, José Luis (1994), Territorios en transición: crítica a la planificación regional en América Latina, Universidad Autónoma del Estado de México, México.

Cortina, Héctor (2006), "Uso del suelo y deforestación en los Altos de Chiapas", tesis de doctorado, Universidad Nacional Autónoma de México, México. 
Entrikin, J. Nicholass (1991), The Betweenness of Place, MacMillan, Londres.

Frémont, Armand (1976), La region: espace vécu, Presses Universitaires de France, París.

Fukuyama, Francis (1989), "The end of History", The National Interest, 16, New Jersey, pp. 3-19.

Giddens, Anthony (1984), La constitución de la sociedad, Amorrortu, Buenos Aires.

Gómez-Mendoza, Josefina (2001), "Un mundo de regiones: geografía regional de geometría variable", Boletín de la Asociación de Geógrafos Españoles, 32, Madrid, pp. 15-33.

Harvey, David (1969), Teorías, leyes y modelos en geografía, Alianza, Madrid.

Harvey, David (2000), Espacios de esperanza, Akal, Madrid.

Hiernaux, Daniel (1991), "En la búsqueda de un nuevo paradigma regional”, en Blanca Ramírez, Nuevas tendencias en el análisis regional, Universidad Autónoma Metropolitana, Xochimilco, México, pp. 33-48.

López, Liliana y Blanca Ramírez (2010), "Pensar el espacio: región, paisaje, territorio y lugar en las ciencias sociales", ponencia presentada en el seminario La noción del territorio y sus revisiones teóricas, Universidad Autónoma Metropolitana, Xochimilco, México.

Massey, Doreen (1978), "Regionalism: some current issues", en Capital and Class, vol. 6, Socialist Economists, Londres, pp. 106-125.

Massey, Doreen (1996), "Politicising space and place", Scottish Geographical Magazine, 112 (2), Scottish Geographical Society, Perth, pp. 117-123.

Massey, Doreen (2005), For space, Sage, Londres.

May, Jon y Nigel Thrift (eds.) (2001), Timespace, Routledge, Londres.

Moncayo-Jiménez, Édgar (2004), Nuevos enfoques del desarrollo territorial: Colombia en una perspectiva latinoamericana, Universidad Nacio- 
nal de Colombia-Red de Estudios de Espacio y Territorio-Programa de las Naciones Unidad para el Desarrollo-CEPAL, Bogotá.

Ohmae, Kenichi (995), "Putting global logic first", Harvard Bussiness Review, Harvard Scholl of Economists, Boston, pp. 119-125.

Pradilla-Cobos, Emilio (1984), Contribución a la critica de la teoría urbana: del "espacio" a la "crisis urbana", Universidad Autónoma Metropolitana, Xochimilco, México.

Ramírez, Blanca (2003a), Modernidad, posmodernidad, globalización y territorio: un recorrido por los campos de las teorías, México, Universidad Autónoma Metropolitana, Xochimilco-Miguel Ángel Porrúa, México.

Ramírez, Blanca (2003b), "Procesos territoriales, escalas y utopías", Ciudades, 60, Red Nacional de Investigación Urbana, Puebla, pp. 9-13.

Ramírez, Blanca (2006), "Espacio-tiempo y territorio", Ciudades, 70, Red Nacional de Investigación Urbana, Puebla, pp. 3-8.

Ramírez, Blanca (2007a), "Escalas territoriales y agentes diferenciales en la integración de políticas de desarrollo", en José Luis Calva, Agenda de desarrollo 2006-2020, t. XIII, Politicas de desarrollo territorial, Universidad Nacional Autónoma de México-Miguel Ángel Porrúa, México, pp. 123-137.

Ramírez, Blanca (2007b), "La geografía regional: tradiciones y perspectivas contemporáneas", Boletín del Instituto de Geografia, 64, Universidad Nacional Autónoma de México, México, pp. 116-134.

Romero-González, Juan (2001), "La comunidad valenciana en la Europa de las regiones", en Juan Romero, Alfredo Morales, Julia Salom y Fernando Vera (coords.), La periferia emergente, Ariel, Barcelona, pp. 7-38.

Rosique-Cañas, José Antonio (2007), "Gestión metropolitana y neoinstitucionalismo: tratando de comprender su problemática”, mimeo.

Terhorst, Pieter (2004), "Continuidades y cambios de los regímenes urbanos: el caso de Ámsterdam”, en José Antonio Rosique Cañas, 
(2007), Gestión metropolitana y neoinstitucionalismo: tratando de comprender su problemática, mimeo.

Tuan, Yi Fu (1974), "Space and place: humanistic perspective”, Progress in Human Geography, 6, Sage, Londres, pp. 233-246.

Wallerstein, Immanuel (1998), "The time of space and the space of time: the future of social science", Political Geography, 1, Elsevier, Londres, pp. 71-82.

Recibido: 18 de enero de 2010. Aceptado: 6 de diciembre de 2010.

Blanca Rebeca Ramírez-Velázquez. Es doctora en urbanismo por la Facultad de Arquitectura de la UnAm; es maestra de planeación regional por la Universidad de Aberdeen, en Gran Bretaña, y tiene un diplomado en ordenamiento del territorio por la Sorbona, París III, Instituto de Altos Estudios de América Latina. Estudió la licenciatura en geografía en la unAm. Pertenece al Sistema Nacional de Investigadores (SNI), nivel II, y es profesora-investigadora del Departamento de Teoría y Análisis, de la División de Ciencias y Artes para el Diseño, en la Universidad Autónoma Metropolitana, Xochimilco. Entre sus funciones profesionales destacan: coordinadora del Posgrado en Urbanismo, Investigación y Docencia de la Facultad de Arquitectura, UnAm, 1986-1992, coordinadora del Área de Investigación y Gestión Territorial del Posgrado en Ciencias y Artes para el Diseño, UAM, Xochimilco, 2002-2008, y miembro de la Junta Directiva de la UAM entre 1998 y 2009. Es fundadora de la Red Nacional de Investigación Urbana y del Grupo Internacional de Geografía Crítica. En la actualidad, sus líneas de investigación son: globalización y procesos territoriales, procesos rurales y regionales, y movilidad laboral en la Región Centro de México. Entre sus publicaciones más recientes destacan: “¿Existe el desarrollo regional/local en el neoliberalismo?”, en Elizabeth Zamora, Raúl Jiménez Guillén, Jaime Ornelas, Carlos Salas y Dídimo Castillo (coords.), El desarrollo hoy en América Latina, El Colegio de Tlaxcala, Tlaxcala, pp. 109-124 (2008); "Retos de la geografía humana en los albores del siglo xxı”, en Martha Chávez, Octavio González Santana y María del Carmen Ventura (eds.), Geografía humana en ciencias sociales: una relación reexaminada, El Colegio de Michoacán, Zamora, pp. 419-440 (2009); "Alcances y dimensiones de la movilidad: aclarando conceptos", Ciudades, 82, Red Nacional de Investigación Urbana, México, pp. 3-8 (2009); "Core-Periphery Models", en R. Kitchein y N. Thrift (eds.), The 
International Encyclopedia of Human Geography, vol. 2, Elsevier, Oxford, pp. 286-291 (2009); "De la escala al espacio en la construcción del desarrollo regional”, en Ramiro Fernández y Carlos Brandao, Escalas y políticas del desarrollo regional, Universidad del Litoral-Miño Dávila, Santa Fe, pp. 217-237 (2010); comp., Formas territoriales. Visiones y perspectivas desde la teoría, Miguel Ángel Porrúa-Universidad Autónoma Metropolitana, México (2008). 